Article

\title{
Land Use Change Ontology and Traffic Prediction through Recurrent Neural Networks: A Case Study in Calgary, Canada
}

\author{
Abul Azad and Xin Wang *(D) \\ Department of Geomatics Engineering, University of Calgary, Calgary, AB T2N 1N4, Canada; akazad@ucalgary.ca \\ * Correspondence: xcwang@ucalgary.ca; Tel.: +1-403-220-3355
}

check for

updates

Citation: Azad, A.; Wang, X. Land Use Change Ontology and Traffic

Prediction through Recurrent Neural

Networks: A Case Study in Calgary, Canada. ISPRS Int. J. Geo-Inf. 2021, 10, 358. https://doi.org/10.3390/ ijgi10060358

Academic Editors: Aaron Costin,

Samad M. E. Sepasgozar,

Sara Shirowzhan and Wolfgang Kainz

Received: 27 February 2021

Accepted: 20 May 2021

Published: 23 May 2021

Publisher's Note: MDPI stays neutral with regard to jurisdictional claims in published maps and institutional affiliations.

Copyright: (C) 2021 by the authors. Licensee MDPI, Basel, Switzerland. This article is an open access article distributed under the terms and conditions of the Creative Commons Attribution (CC BY) license (https:// creativecommons.org/licenses/by/ $4.0 /)$.

\begin{abstract}
Land use and transportation planning have a significant impact on the performance of cities' traffic conditions and the quality of people's lives. The changing characteristics of land use will affect and challenge how a city is able to manage, organize, and plan for new developments and transportation. These challenges can be better addressed with effective methods of monitoring and predicting, which can enable optimal efficiency in how a growing city like Calgary, Canada, can perform. Using ontology in land use planning is a new initiative currently being researched and explored. In this regard, ontology incorporates relationships between the various entities of land use. The aim of this study is to present Land Use Change Ontology (LUCO) with a deep neural network for traffic prediction. We present a Land Use Change Ontology (LUCO) approach, using expressions of how the semantics of land use changes relate to the integration of temporal land use information. This study examines the City of Calgary's land use data from the years 2001, 2010, and 2015. In applying the LUCO approach to test data, experimental outcomes indicated that from 2001 to 2015 residential land use increased by 30\% and open space decreased by $40 \%$. Forecasting traffic is increasingly essential for successful traffic modelling, operations, and management. However, traditional means for predicting traffic flow have largely assumed restrictive model architectures that have not controlled for the amounts of land use change. Inspired by deep learning methods and effective data mining computing capabilities, this paper introduces the deep learning Recurrent Neural Network (RNN) to predict traffic while considering the impact of land use change. The RNN was successful in learning the features of traffic flow under various land use change situations. Experimental results indicated that, with the consideration of LUCO, the deep learning predictors had better accuracy when compared with other existing models. Success of our modeling approach indicates that cities could apply this modeling approach to make land use transportation planning more efficient.
\end{abstract}

Keywords: Land Use Change Ontology (LUCO); Recurrent Neural Network (RNN); traffic prediction

\section{Introduction}

It is believed that urbanization has generated several economic, environmental, and social issues that must be addressed when planning for traffic management. The current technological innovation era has led urban planners and decision-makers to consider data analysis and data management when addressing traffic prediction. In the past decade, Calgary has become one of the fastest growing cities in Canada [1]. Over the past three decades, Calgary's steep economic and urban progress has resulted in it becoming a metropolis of 1.3 million residents. However, with growth comes a host of issues, such as traffic congestion, lack of municipal utilities, and shortage of housing needs.

Researchers have identified a relation between land use change and traffic prediction. There are various models currently employed to model land use changes and traffic prediction [2,3]. However, there is a continuous necessity for new approaches to re-evaluate current models and improve them. GIS and remote sensing techniques make it possible to collect vast amounts of data about the Earth's surface at a finer resolution. Detection of land use change is one of the key research areas of GIS. Although several techniques $[2,4,5]$ 
can accurately detect land use changes from GIS data, there exist great challenges and opportunities to effectively organize and model such information from multi-temporal data.

Traditional acquisition of land use and supporting data is a complex process $[6,7]$. Historic and recent land use data are prerequisites for urban planners to predict and simulate the future status of an urban system, especially in the practice of integrated land use and transportation modeling systems. Unfortunately, to date, no effective and practical ontology has been presented to extract land use information and utilize it efficiently to predict traffic.

Besides their social, demographic, and economic implications, land developments also have a profound impact on the surrounding transportation system. Whether residential or commercial, new developments inevitably result in an increase in all types of vehicular activity [8]. The primary impact of a land development is travel demands generated directly by users of the development, and the corresponding traffic flows. This can be defined as direct transportation impact of land development. The new land development may also have an indirect effect on the host community and the nearby area traffic [8].

An analysis of the traffic attributed to a development would require the use of land use analysis (to quantify land use change) and transportation demand analysis (to quantify the impact of land development on traffic flows). However, these forms of methodology are not successful in demonstrating the ability to determine the impact of traffic on the development. Due to the inefficacy of these methods, it could be determined that there was a need for further research in creating an analytical framework and methodology that would focus on the interactions between transportation and land use as they relate to influencing a development and the resulting induced travel demand [9].

Land use change research has substantially improved the semantic interoperability among existing land use classification systems [10-12]. Historically, studies regarding land use change focused on matching the conceptual definition of land use types rather than on the specific purpose of predicting traffic. The semantic modeling of the spatiotemporal changes in regional traffic prediction has not been studied so far. Thus, the purpose of this paper is to address this issue.

Predicting traffic volume is of great importance when seeking to optimize the management of traffic of an urban area. Methods used to solve traffic volume prediction have traditionally used a parametric approach. Even though the deep learning approach has become more popular, there are those who continue to explore traffic prediction using conventional methods. Researchers remain interested in their use of both parametric and nonparametric methods, such as using regression and neural networks, respectively.

The use of ontology in land use planning is currently being researched and explored. The term 'ontology' originates from the Greek words onto (being) and logia (written or spoken discourse). Ontology is a concept that deals with the first principles or the essence of things [13]. In this study, ontology is the formal specification of concepts and relationships for a particular domain [14]. In land use planning, ontology encompasses a representation and definition of the groups' specific land uses. It also incorporates relationships between the types of land [10]. Ontology can provide interesting results when comparing various types of land use.

Because of recent advantages in Geographic Information System (GIS)-based land use, traffic data, and aerial imagery, it has become an effective option that reveals characteristics of land use changes and predicts traffic volume. A wide variety of GIS-based methodologies have been proposed in the literature to analyze a city's land use changes and traffic estimations [2,15-17].

Although there has been significant progress in land use ontology in recent years, ontology-based outcomes are still not used in deep learning for traffic prediction. The reason we utilize ontology is that it can help us generate meaningful land use change that we can use in deep learning neural network for traffic prediction. To the best of our knowledge, this approach is the first work to combine land use change ontology and deep learning for traffic prediction. It can be applied to other land use and transportation planning domains. 
Several factors were considered when examining land use, which included new developments, infrastructure, and redistricting. Understanding this progression and identifying new trends or patterns is advantageous to practitioners. The proposed approach is called Land Use Change Ontology (LUCO), in that it models land use progression, which we have adapted from Harbelot et al. [12]. The purpose of this study was to measure land use geometrically and to identify changes in attributes while implementing an ontologybased system. The LUCO model can identify the changes that typically contribute to variations in traffic prediction in specific areas.

Artificial Neural Network (ANN) is a computational model focused on the structure and functions of biological networks [18]. Deep learning is part of a broader group of machine learning methods based on ANN [19]. The term 'deep' in deep learning comes from the use of multiple layers in the network. In deep learning, the layers are also allowed to be heterogeneous and deviate widely from the biologically informed connectionist's models. The primary purpose of having these layers is to make the model more efficient. The application of deep learning has recently become a thriving field of study with many practical applications and active research topics. When using ANN, the results showed that temporal data typically exhibit challenges, such as over fitting or nonlinear relationships, as well as redundancy dependence between multiple datasets [19]. Deep learning methods allow computers to learn from input data and understand the world in terms of a hierarchy of concepts [19]. Because of Deep Neural Network's (DNN) ability to learn a hierarchy of concepts, it was of interest to focus on how the neural network functions affect land use changes over time while including temporal data to predict traffic. In this study, a novel approach was introduced by combining Land Use Change Ontology and the deep neural network RNN, in order to improve traffic prediction.

Land use change have become an important part of today's transportation planning. The success of traffic prediction depends on many factors, among which land use change, traffic demand, and the number of traffic in a specific area during the next period of time are vital. Accurate traffic prediction can lead to remarkable benefits. For instance, it allows transportation planner to identify traffic congestion areas. From a broader point of view, land use change and traffic prediction also help to optimize traffic efficiency via improving the imbalance of transport capacity across the city.

This research work explored the following research questions:

- What was the land use change ontology (LUCO) in Calgary and how did LUCO measure land use changes?

- How did different deep learning and data mining methods use this land use change to predict traffic?

- How to evaluate the deep learning and data mining methods when wanting to predict traffic?

To explore these research questions and issues, this study proposes a framework based on land use change ontology and traffic prediction. This paper makes notable contributions, summarized as follows:

- To identify Calgary's land use changes using an ontology (LUCO) model.

- To use land use changes as a key variable in a deep learning RNN model to predict traffic in Calgary.

- To provide detailed statistical analysis based on the relationship between land use change and traffic prediction.

- To provide verification of the validity of this approach and possibility for future work.

To the best of our knowledge, we are the first to use land use change ontology and deep neural networks to deal with land use change and traffic prediction. Different from the other methods, we used Calgary's data as a case study in order to assess the influences between land use changes and deep neural network in predicting traffic. In this paper, we propose that LUCO be linked to a temporal traffic prediction model based on a collaborative learning technique using the deep learning Recurrent Neural Network 
(RNN). Empirical experiments were conducted to explore these contributions. Section 2 of this paper describes the relevant research in ontology and traffic prediction. Section 3 introduces the methodology and procedures of the proposed LUCO and RNN models. Subsequently, Section 4 describes the experimental results of how the model was able to gradually increase knowledge about land use changes and its application in traffic prediction. Finally, Section 5 presents the conclusions and areas for future research.

\section{Related Works}

There have been several attempts to evaluate changes in land use patterns using GIS and remote sensing imagery. These studies tended to put their emphasis on developing a model that look at the changes in space, time, and context. One of the key advancements in research has been the discovery of GIS ability to detect land use changes. This has successfully been applied to a wide range of geospatial applications, such as landscape monitoring, natural resource estimation, and environmental management [20]. Although several GIS techniques $[10,21]$ can detect land use changes, numerous challenges continue to exist on effectively organizing and developing this type of information.

\subsection{Land Use Change Ontology}

The goals of land use change ontology include developing different classification systems through semantic analysis. This strategy, known as ontology matching or alignment, requires the calculation of the semantic similarity amongst varying land use class definitions. These classification systems include geometric, feature-based, network, alignment, and informationtheoretic modeling [12]. Because a land type definition was usually given in a text block, text analysis techniques were also explored for extracting the spatial and nonspatial attributes of land use classes, which augment the formal definition in the ontology [12].

There are few studies that have focused on land use change semantic modeling only [10]. This is due to the complexity in modeling spatial objects, which contain four distinct elements: (a) geographical location; (b) geometric shape or size; (c) topological intersections with other spatial objects; and (d) temporality. Beyond this, modeling complexity increases dramatically when a change process involves combining multiple dimensions, such as traffic prediction from land use change.

Harbelot [12] laid the theoretical foundation for representing changes in a spatial object. The recently developed spatiotemporal data model LC3 (Land Cover Change Continuum) revealed land use changes. Numerous change processes, including splitting, merging, and annexation, were defined in the LC3 model, which make it superior for modeling land use change information; however, all of the topological relationships, such as "contain" and "within", were predefined in the ontology [12]. This caused issues in the modelling and querying of large numbers of spatial objects; this approach also has not been utilized in predicting infrastructure such as traffic.

Hyvonen et al., [22] presented a spatio-temporal model to analyze change types in historic regions, such as countries, municipalities, and cities. Over time, regions can be renamed, merged together, split into parts, and annexed or moved to and from other regions. The model was able to detect some patterns based mainly on spatial criteria and semantic representations to represent name changes. However, these patterns did not clearly refer to real-world phenomena as there is a lack of domain knowledge considered in the analysis using ontological hierarchies and structures.

Former studies have used various semantic approaches to focus on the spatial structure of land use. Yang et al. [23], for instance, used these approaches to model urban spaces and road networks. Another example is Balestrieri [24], who used graphs to model the structure of a road network territory. These studies generally have omitted the temporal dimension, which requires additional modeling procedures for traffic prediction. To overcome these limitations in land use changes and spatiotemporal semantic modeling, this paper proposes the development of a context to address how land use changes contribute to traffic prediction. 
In this work, we proposed a novel methodology that combined ontology and deep learning techniques by providing a framework to predict traffic. Our model extended the land use change ontology to a well-used deep learning method, Recurrent Neural Network. The reason we utilized ontology was that it could help us generate land use change, which was particularly important for traffic prediction. Another crucial reason was that deep learning architectures, such as the RNN, are a better representation of temporal traffic prediction. With respect to the proposed state-of-the-art system, the main advantages were the ontology's ability to handle complex land use change situations and the ability to further extend an entirely deep learning-based system that did not require hand-crafted rules for traffic prediction.

Urban land use patterns, including land use function, land development intensities, and layout, work together to represent the traffic demand's spatial and temporal characteristics and traffic flow. Reasonable land use will maximize the total demand of urban traffic trips and make trip distributions more uniform while easing traffic congestion. It would be beneficial to city planners to be able to forecast traffic relating to the land uses, which would greatly assist in resolving any traffic issues or possible congestions [25].

\subsection{Traffic Prediction}

A substantial amount of work has focused on increasing the ability to interpret the dynamics of traffic prediction. Nevertheless, the most common methods, such as AutoRegressive Integrated Moving Average (ARIMA) and its sub-categories, have been found to be restrictive to only obtaining information that reveals time series traffic predictions [26] Alternatively, hybrid models that take advantage of two or more models were found to be more successful. In the combination of maps and ARIMA for traffic prediction at different time intervals, the results showed an improvement over the use of a single model, such as ARIMA [26]. Another widely used approach was the Kalman Filter. Okutani and Stephanedes introduced the Kalman Filter for predicting traffic, with improved performance [27]. Although using this method provided robust results from the fluctuating data, it also created concerns, such as skewed outcomes.

All the previously described methods have concerns with non-linearity. Traffic prediction has non-linearity characteristics that have to be considered. It was thought that nonparametric Neural Networks (ANN) could address these concerns. Nikravesh et al. [28] explored the correctness of Multi-Layer Perceptron (MLP), which is an architecture of ANN, for predicting traffic. Their results showed that MLP was more accurate than another model, Support Vector Machine (SVM). Liu et al. [29] applied Local Stacked Auto-Encoders (LSAEs) and Global Stacked Auto Encoder (GSAE) to obtain local traffic prediction and subsequently used Long Short-Term Memory (LSTM) to predict traffic. Authors observed that LSTM could be an efficient approach to in solving nonlinear problems, as long as they contained a sufficient number of components. Nevertheless, if the number of variables became very large, the amount of computations became complicated. In this situation Deep Neural Network (DNN) provided better results. DNN had at least two or more hidden layers relating to ANN [30]. Having more layers in neural networks could provide better results from inputs. Ateeq et al. [30] applied DNN to predict delays in traffic while Hua et al. [31] investigated the traffic prediction with deep learning and Random Connectivity LSTM, based on the standard LSTM.

Recently, there have been more popular deep learning-based traffic prediction studies that have become available in the literatures; e.g., Zhang et al. [32] examined the deep learning traffic prediction model based on a convolution neural network (CNN) framework. An LSTM recurrent neural network is popular for dealing with time-series data [29]. Zhou et al. [33] proposed a multi-scale and high-precision LSTM traffic prediction model. This model was used to predict short-term traffic flow data, and various predictions were analyzed by the combination index. Their results demonstrated that the model was able to perform better when compared with other existing approaches. Wu et al. [34] studied a deep learning framework for traffic prediction by analyzing LSTM to address the 
temporal correlations in traffic data. Narmadha and Vijaykumar [35] revealed multivariate traffic prediction by using LSTM in their study. LSTM model results were compared with convolution neural network (CNN) models. It was found that the deep learning LSTM could be used in several learning and prediction scenarios.

Victorovic et al. [17] proposed Connected Traffic Data Ontology to provide a more suitable solution for time-sensitive data coming from multi-sensory platforms. Recent publications also showed that some ontologies have been developed in this domain [36-38]. Since this approach requires integrating cross-domain data, it is important to follow at least general guidelines in preparing such an ontology. Nevertheless, there is no standardized methodology to do this as yet.

There has been little research with ontology focusing on land use change and traffic prediction to our best of knowledge. Approaches have varied in these studies, from the development of entity-based ontology to the domain-specific ones [17]. However, this involved a very detailed set of ontology, which showed a very context-specific and isolated structure. Furthermore, it did not leave much space for integration with other methods, such as deep learning. Therefore, to develop an ontology for the integration of heterogeneous, different-type, real-world traffic data, deep learning neural networks in particular seemed to be a better way to proceed.

Deep learning methods can be combined with land use change methods to learn the feature representations of traffic prediction. These methods provide flexible and expressive models that, if designed and trained properly, can easily outperform parametric and statistical methods. The additional parameters of land use can also provide a way to incorporate additional data as phase-timing information. There are still considerations to be explored and much room for improvement, especially in the realm of traffic prediction.

\section{Datasets and Methods}

\subsection{Study Area and Data}

Calgary is a city located in the mid-southern part of Alberta, Canada, with a population of 1.3 million people, covering an area of $800 \mathrm{~km}^{2}$ [39]. In addition to being the hub of Canada's primary oil industry, it is recognized as being the political, economic, financial, cultural, educational, and transportation center of Alberta. The ongoing growth in Calgary has very much been influenced by several industrial initiatives that have impacted the ever-evolving patterns of land use over time. As a result of the large-scale growth, an efficient, large-scale monitoring system was essential in creating a sustainable means for transportation planning. The northeast portion of Calgary (see Figure 1), which has an area of $120 \mathrm{~km}^{2}$, was selected as an example in order to meet the goals of this study.

The City of Calgary identifies major categories of land uses, including commercial, industrial, major infrastructure, and open space. Three types of residential land use are also classified based on the City of Calgary's data sources-residential high, medium, and low density. This study generalized the land use type according to City of Calgary's land use classifications.

Traffic data were collected in the abovementioned area from the years 2000 to 2015. The years were selected based on the availability of GIS-based data from the City of Calgary. The average annual monthly traffic data provided the numbers for vehicles passing a traffic observing location over a 24-h time span, on an average weekday. For sampling, the study adopted a prediction evaluation technique referred to as walk-forward validation or backtesting. Conventional evaluation methods, such as k-fold cross-validation, were unsuitable for use in time series data because they did not consider the temporal or sequential factor $[9,40]$. Seventy percent of the data were selected as the training set while rest of the data was used for the testing models. All the available data were incorporated into the preparation of the models to ensure better accuracy. Land use data were in years interval and traffic data were monthly; this requires data integration. For data integration, the most common method, the Denton-Cholette without an indicator, was used in this study. It performed a simple interpolation that met the temporal additivity constraint [41]. 


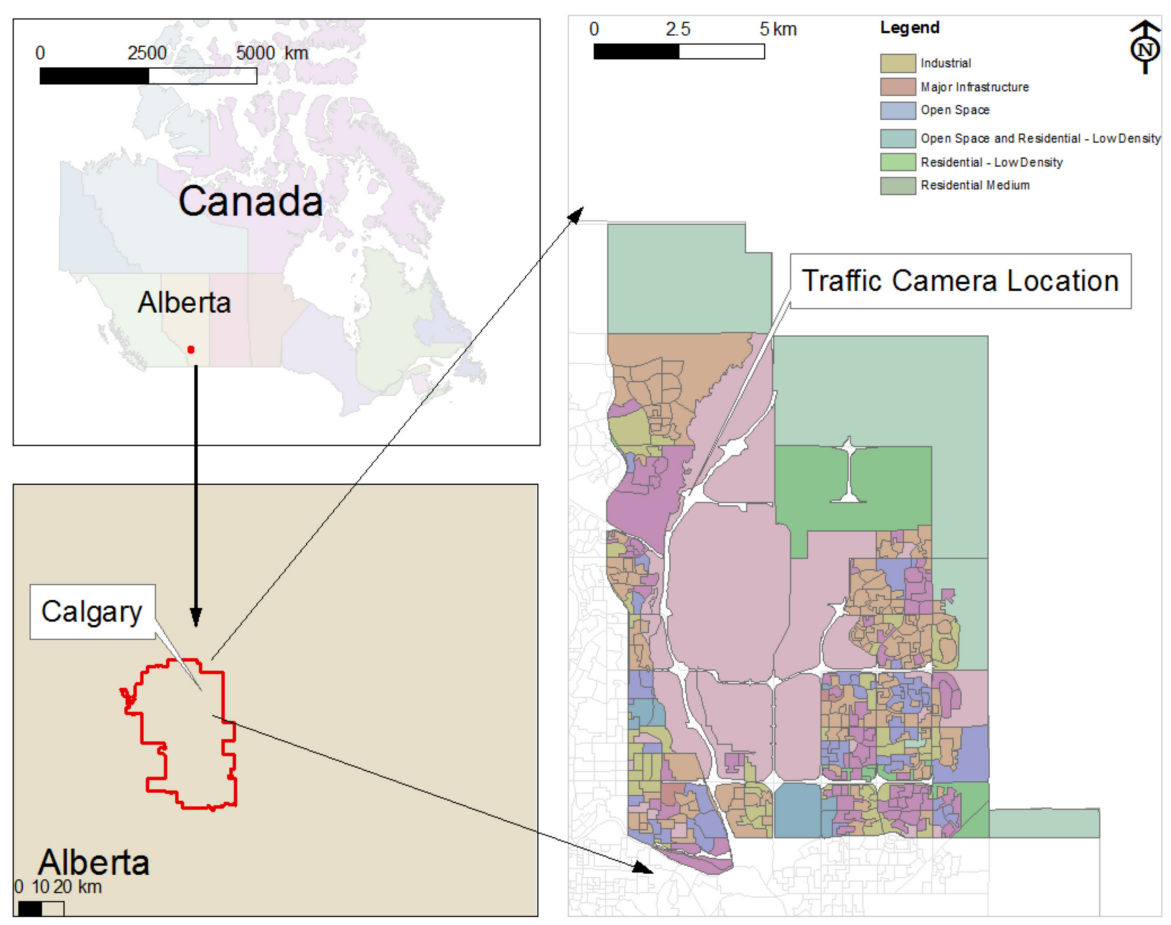

Figure 1. The geographic study area: northeast Calgary.

The original land use datasets were obtained from the University of Calgary, derived from the City's Land Use Bylaw Code classification of Urban Land Use and Planning Standards of Development Land. The designated land use classes are shown in Table 1.

Table 1. This table is based on the "Land Use Bylaw Code" for Classification of Urban Land Use and Planning Standards of Development, City of Calgary.

\begin{tabular}{|c|c|}
\hline Land Use and Other Variables & Brief Description \\
\hline Commercial & $\begin{array}{c}\text { Community Commercial } \\
\text { Commercial-Core } \\
\text { Auto-Oriented Commercial Corridor } \\
\text { High Rise Office and Mixed Use } \\
\text { Local Commercial } \\
\text { Mixed Pedestrian/Auto-Oriented Commercial } \\
\text { Corridor } \\
\text { Pedestrian-Oriented Commercial Corridor } \\
\text { Regional Commercial }\end{array}$ \\
\hline Industrial & $\begin{array}{l}\text { Heavy Industrial } \\
\text { General Industrial } \\
\text { Industrial Interface } \\
\text { Industrial Storage }\end{array}$ \\
\hline Residential & $\begin{array}{l}\text { Residential Medium Density } \\
\text { Residential Low Density }\end{array}$ \\
\hline Major Infrastructure & $\begin{array}{l}\text { City Regional Infrastructure } \\
\text { Transportation and Utility Corridor }\end{array}$ \\
\hline Open Space & - \\
\hline Aerial Imagery & Aerial Imagery Year 2001, 2010, 2015 \\
\hline Temporal Traffic Data & $\begin{array}{l}\text { Traffic data (aggregated) collected from different } \\
\text { monitoring stations northeast of Calgary from year } \\
2000 \text { to } 2015\end{array}$ \\
\hline
\end{tabular}




\subsection{Methodology}

This study proposes a novel hybrid Land Use Change Ontology (LUCO) and deep neural network model for traffic prediction. Figure 2 illustrates the overall methodology of this study. It contains two interrelated major sections. The first section explains the procedures of Land Use Change Ontology. The first section of the overall methodology proposes the LUCO model using the Geographic Information System's (GIS) spatial capabilities to explore land use changes. The LUCO's properties provided a representation of the progression of these changes. This model is selected to represent the conditional relationship between sequential or temporal land use changes that are connected to an individual type of land use. While the second section details the use of Recurrent Neural Network in predicting traffic. The overall methodology is described in following two subcategories.

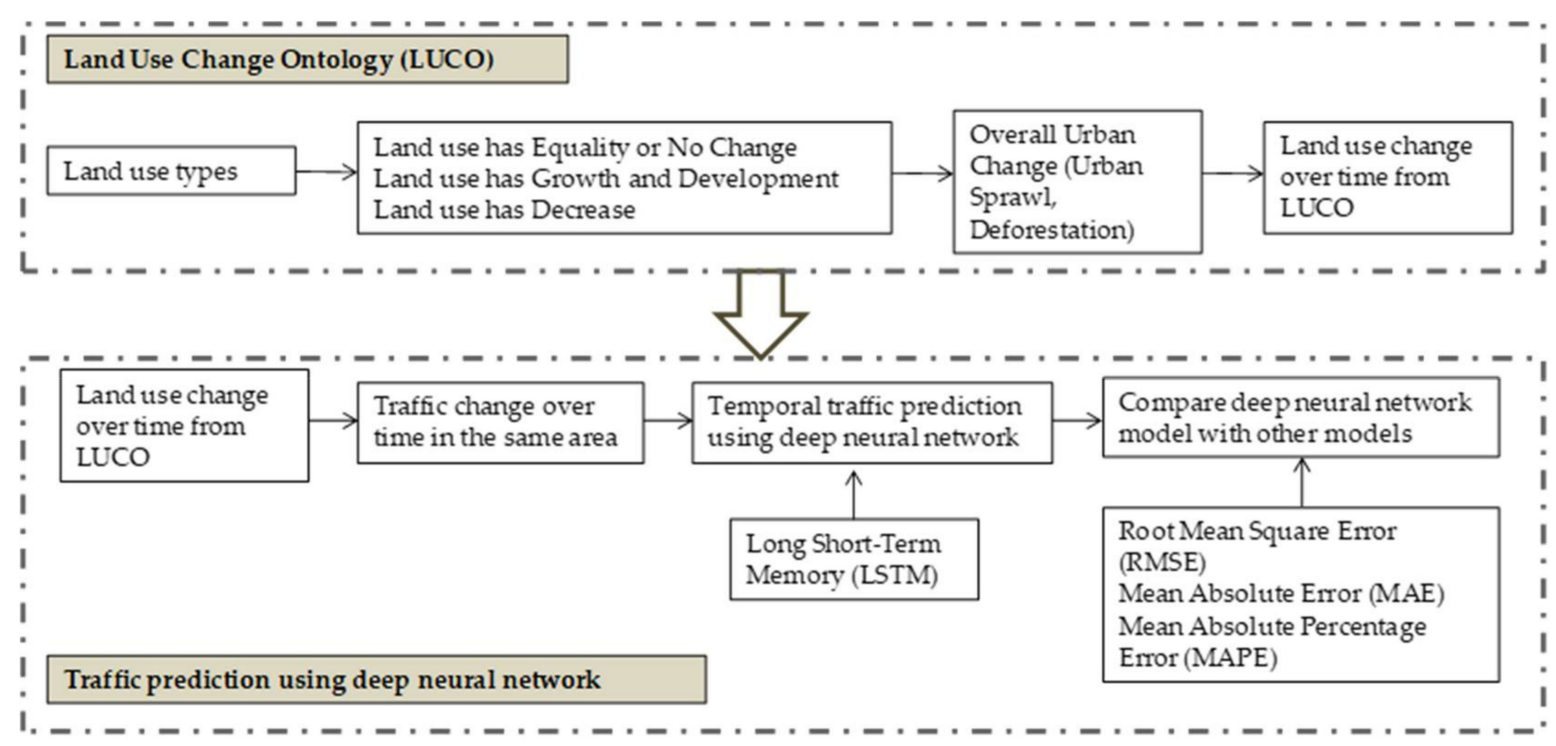

Figure 2. Overall methodology.

\subsubsection{Land Use Change Ontology (LUCO)}

In one or more dimensions, land use change could cause a spatial land use-type feature to split into multiple types and merge with other spatial types or dissolve into a new type. Furthermore, changes that combined multiple relationships (dimensions), such as these, were difficult to model. Figure 3 illustrates three key challenges facing any semantic model for change information modelling from time series GIS data.

\section{Land use through time}

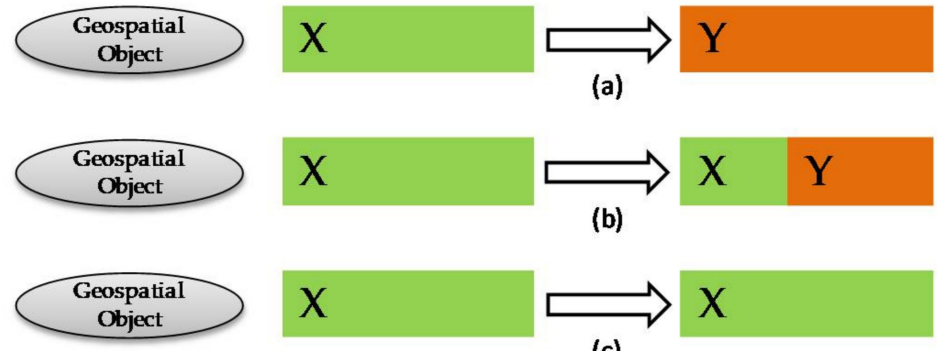

Geospatial land use has changed over time entirely from 2001 to 2010

Local land use type changed but globally uniform object between 2001 and 2010

No land use change within the certain time period 2001 to 2010

(c)

Figure 3. Land use change challenges. (a) represents a change of land use for the entire region; (b) represents local land use change of a region; (c) represents no changes in land uses within a certain time period. 
In Figure 3a, note that the entire geospatial land use of the study area has changed over time. The geospatial objects demonstrated two distinct land use attributes at different timestamps: land use type $X$ in 2001 and land use type $Y$ in 2010. Semantic models were needed to integrate both definitions of this object. For the second case (Figure 3b) local land use type changed within a globally uniform object. The geospatial object only contained attribute $X$ in 2001, but it was mostly maintained with partial element $Y$ changes in 2010. When forming a semantic model for the elements of this geospatial object, it was hard to classify land use types, as it could reasonably be described as $\mathrm{X}, \mathrm{Y}$, or even $\mathrm{X}$ and $\mathrm{Y}$ concurrently (the case in Figure $3 b$ ). The example in Figure $3 b$ could be a land use Separation or Split. In this case, the parent entity continues to exist; however, its geometry created a new geometry corresponding to a new entity. It has a Separation relationship similar to a Split relationship with the difference that the original entity remains [12]. In the third case (Figure 3c), there were no changes in land uses within a certain time period.

To address these issues, a spatiotemporal change detection method was used in detecting changes (where they occur), semantically defining the changes (what they are) and effectively tracking the changes (how they evolve). This required a modelling approach that could store and capture all of these dimensions of information [12]. To model this complexity, and to effectively manage the land use change information from time series geospatial data, this study proposed an integrated computational semantic concept model, LUCO.

Figure 4 illustrates the proposed LUCO model's mechanisms for individual land use types. This model was proposed to evaluate the land use changes that occurred in spatial land use types. These changes were characterized by spatial variations and relate to position or spatial land use footprint. The proposed LUCO was integrated and categorized land use by space (i.e., topological relations), time (Allen Relations [42]), and its semantics identity.

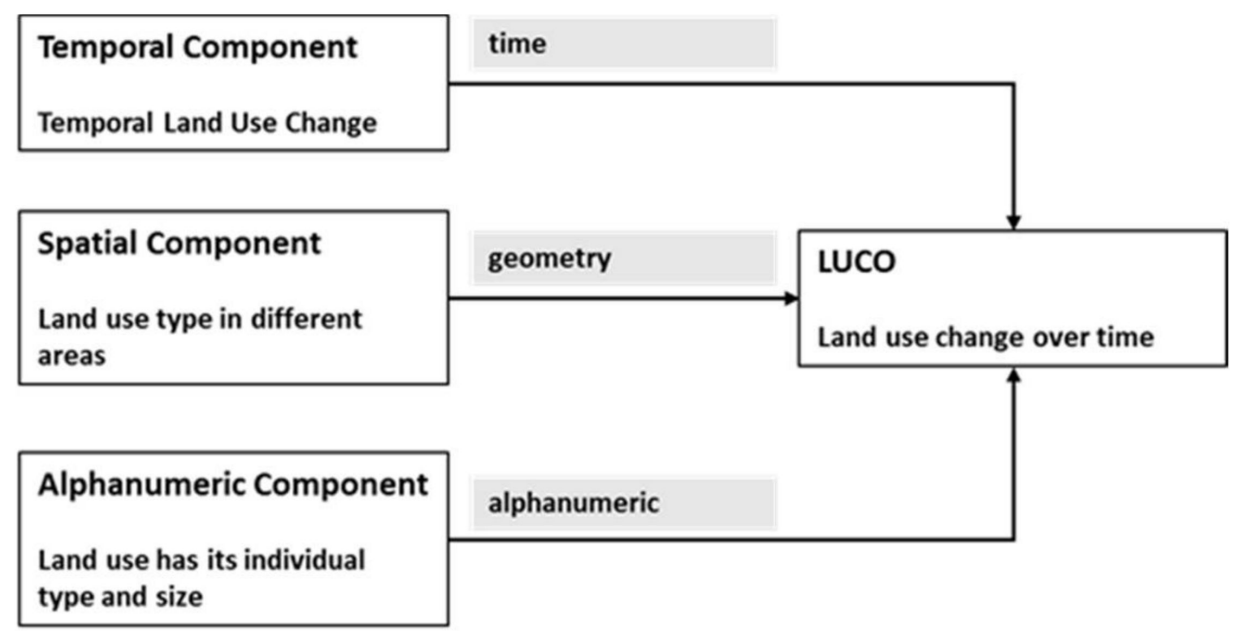

Figure 4. Mechanisms and components of the LUCO model.

To concentrate on the conditional relationships between the varying types of land use, discrete categories were identified to distinguish the factors that impact changes. Thus, land use filiation relationships were divided into the following relationships (Table 2).

The spatiotemporal data of Calgary's northeast quadrant were more likely to change as the city expanded. Therefore, the data integration was a fundamental component of the LUCO model. Most of the land use information systems were designed in order to structure, manipulate, and search data. Additionally, LUCO was able to establish rules for maintaining data integrity. The proposed LUCO represents a spatial object with static properties (i.e., properties of the land use from which a geospatial object was obtained), dynamic properties (i.e., properties that change information over time), and its relationships with other nearby spatial objects. This spatial object formally represents LUCO in that it provides a semantic relationship model. Built upon change annotations, 
this LUCO uses four core components: property, relation, rule, and restriction, as seen in Figure 5 as the first tier of the ontology. Besides the four core components, entities in orange and green represent the first-level and second-level subclasses, respectively. For example, "property" is further divided into "spatial property", "temporal property", "image property", and "data source property". These are explaining the spatial and temporal property of an object, as well as the characteristics of the aerial imagery from which the object was extracted. LUCO's rules provided support for logical reasoning based on reference knowledge from various sources. Our semantic model supported two types of reasoning for detecting spatiotemporal change: logical reasoning and spatial reasoning. Logical reasoning utilized rules specifically defined for making inferences on the change in land use within a geographical area.

Table 2. LUCO filiation relationship description.

\begin{tabular}{|c|c|}
\hline LUCO Filiation Relationship & Description \\
\hline has equality & No change in land use type data \\
\hline has conversion & $\begin{array}{c}\text { No change in the size of the area but a change in the land use } \\
\text { type (example: total area change from residential } \\
\text { to commercial) }\end{array}$ \\
\hline has growth & $\begin{array}{c}\text { No change in land use type but an increase in the size of the } \\
\text { land use of the same type (example: total area increase in the } \\
\text { same residential land use) }\end{array}$ \\
\hline has reduction & $\begin{array}{c}\text { No change in land use type but a decrease in the size of the land } \\
\text { use of the same type (example: total area decrease in the same } \\
\text { residential land use) }\end{array}$ \\
\hline
\end{tabular}

In the ontology, different entities were assigned different prefixes to refer to the namespace to which they belonged. For example, spatial_property, image_property, temporal_property, and thematic_property indicated the spatial, image, temporal, and thematic properties of a spatial object. For instance, "property" is additionally divided into "spatial property", "temporal property", "aerial image property", and "data source property. The "rule" component described the inference ruled in a machine-understandable format, and it was categorized into a reflective or a symmetric to support semantic reasoning. The "polygon" class is a type of "spatial location" class to depict an area property. They were implemented as the predicates in the triple structure: <subject, predicate, object> in OWL [43]. External data for this ontological model came from the literature, remote sensing image, and metadata databases.

Time series aerial imagery was preprocessed using Object-Based Image Analysis (OBIA) techniques to generate the land use classification maps [44,45]. ECognition was used to segment each aerial image at multiple scales (a higher scale and a lower scale) [7]. The mean and standard deviation of image regions were also used for land use classification. After receiving the original classification results that were generated from ECognition, the errors were manually corrected based on the results from higher-scale segmentation. Note that since the focus of this work was focused on incorporating semantic modeling and a deep learning neural network, manual work and the City of Calgary's land use classification were used. This was supported in the land use classification process to remove uncertainties and ensure accurate classification results.

The product generated from the above procedure was a raster map, and it was then vectorized to generate a polygonal file. These were more specifically represented in the subdivisions of the study area according to their land use types. These vector data were compiled into ESRI [46,47]. The dataset was composed of two parts: the spatial part that recorded the geographical periphery of each polygon object and the alphanumerical part that recorded the attributes, such as land use type, years of a spatial object, and related information, integrated from other data sources. 


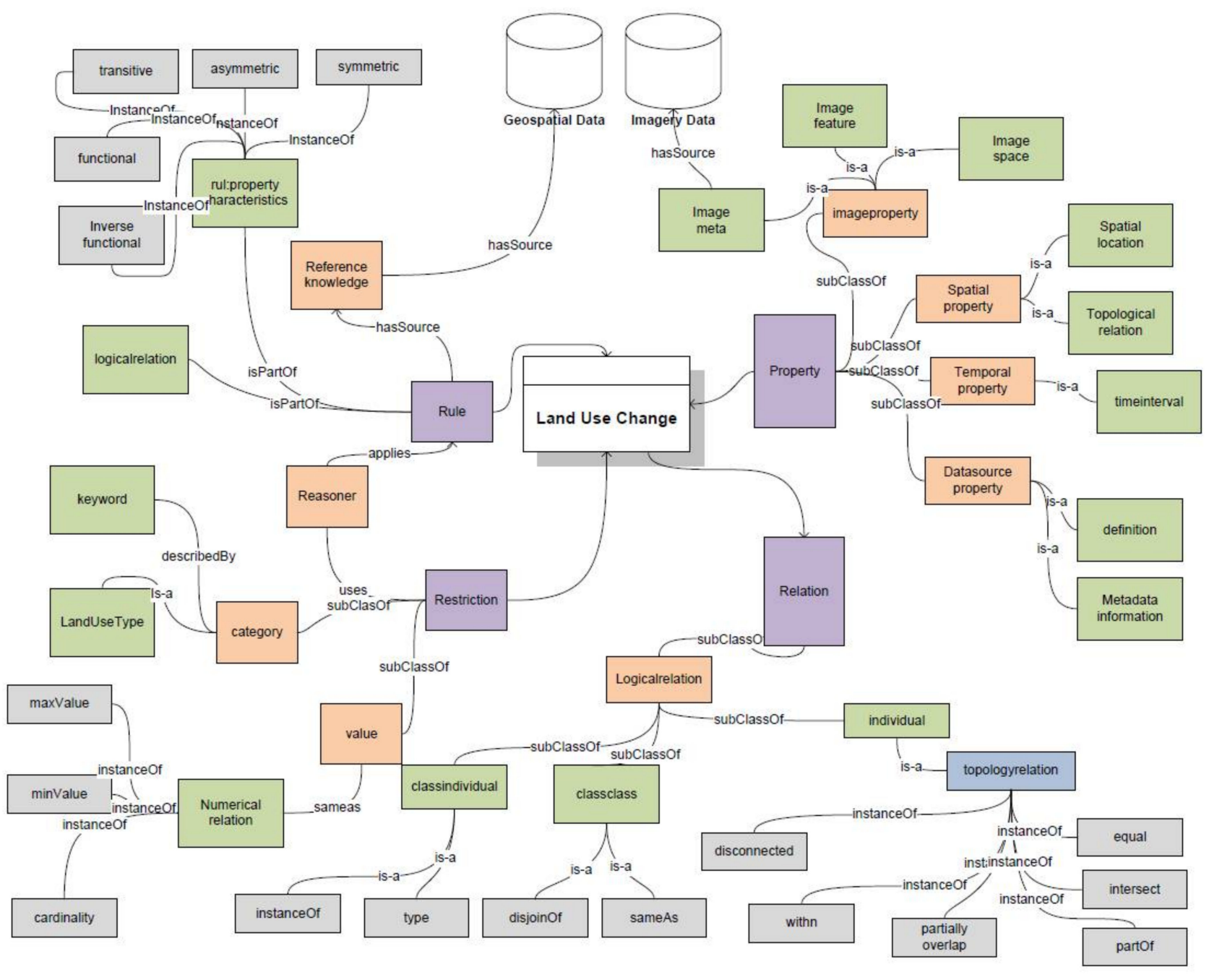

Figure 5. LUCO for land use change adapted with permission from [11], (2016, Li, W.). Nodes of the same color refer to the same level.

The LUCO model provides land use change information over time and space. This section describes the LUCO's framework and how it relates to traffic prediction in northeast Calgary, Canada. LUCO illustrates continuous relationships between land use changes. Continuation derivation is involved in the land use change evolution of a single entity (1-1 relationship) or spatial structures, involving several entities ( $0-n$ or $n-0$ relationships), such as division and merging patterns.

Detecting land use changes is a part of LUCO's process. Given two overlapping land uses correspond to two time positions (tseg), the rules we get are as follows:

Growth: tseg1 is within tseg2;

Reduction: tseg1 contains tseg2;

Equal: tseg1 is equal to tseg2.

The semantic query carried the land use change; the ontology was established in a machine-understandable way. The dataset was then composed of two parts: the spatial part that recorded the geographical boundary of every polygon object and the alphanumeric part that recorded the attributes, such as land use type, year of land use existence and related information, integrated from other data sources. Subsequently, the land use data were loaded from the backend data repository or shapefiles using the GeoTools Feature API [48]. Data records were serialized into triples and written into the triple store of Jena by applying the Jena API [48]. The logical rules defined in this section were also imported into the Jena data store. 
To detect land use change, this study obtained change objects with land use type annotations in 2001,2010, and 2015. The study created a geospatial ontology according to the LUCO structure demonstrated in Figure 5 to model the change objects and their LUCO in the case study area.

Besides the class definition in Figure 5, the study also defined two properties: object property and data property. The object property was mainly used to connect a subject and object, which were both individuals of a specific class in a <subject-predicate-object $>$ triple structure. As shown in the following triple example and Figure 6, we defined various topological relationships as object properties representing the relationship between land use changes.

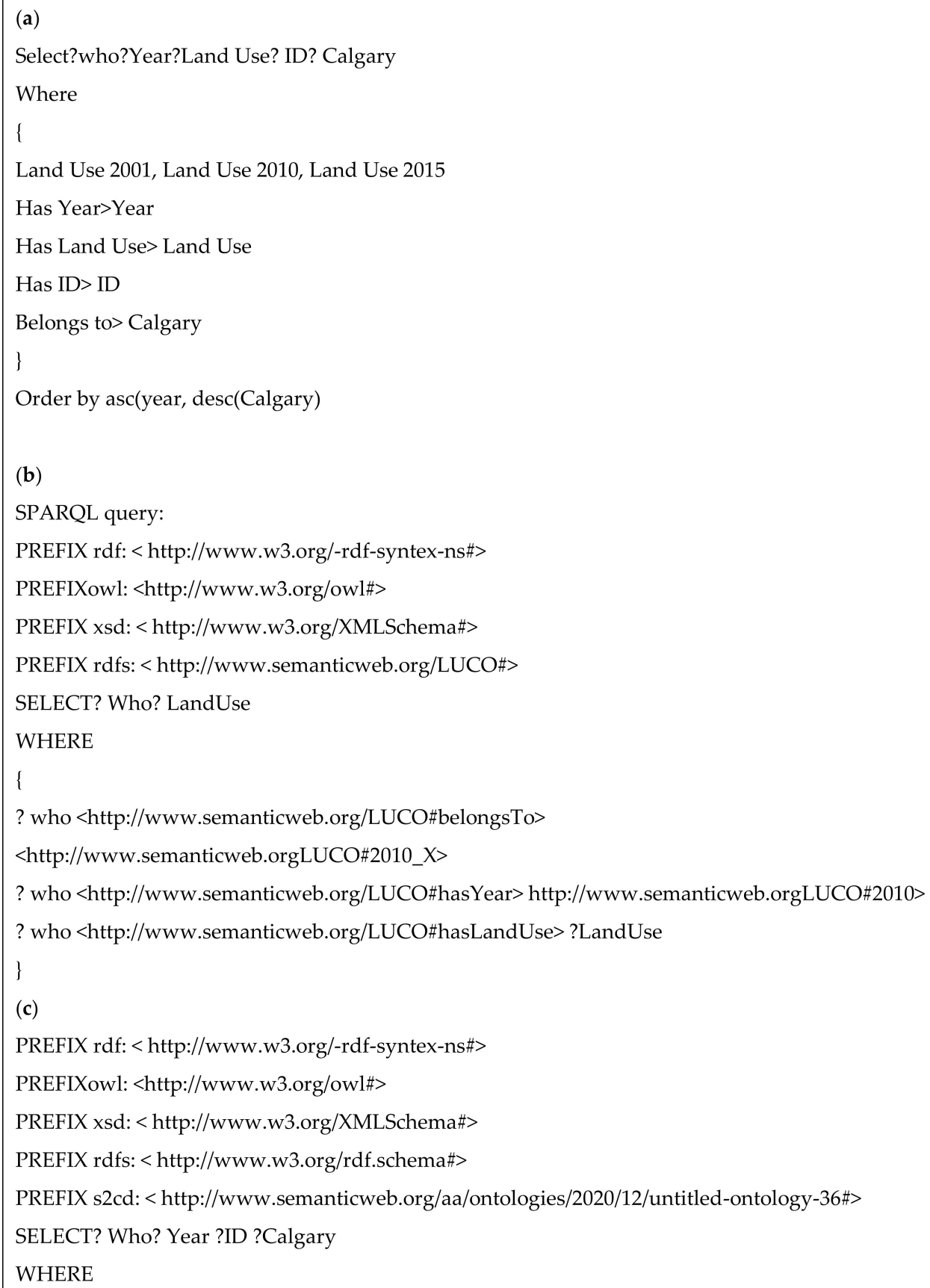

Figure 6. Conts. 


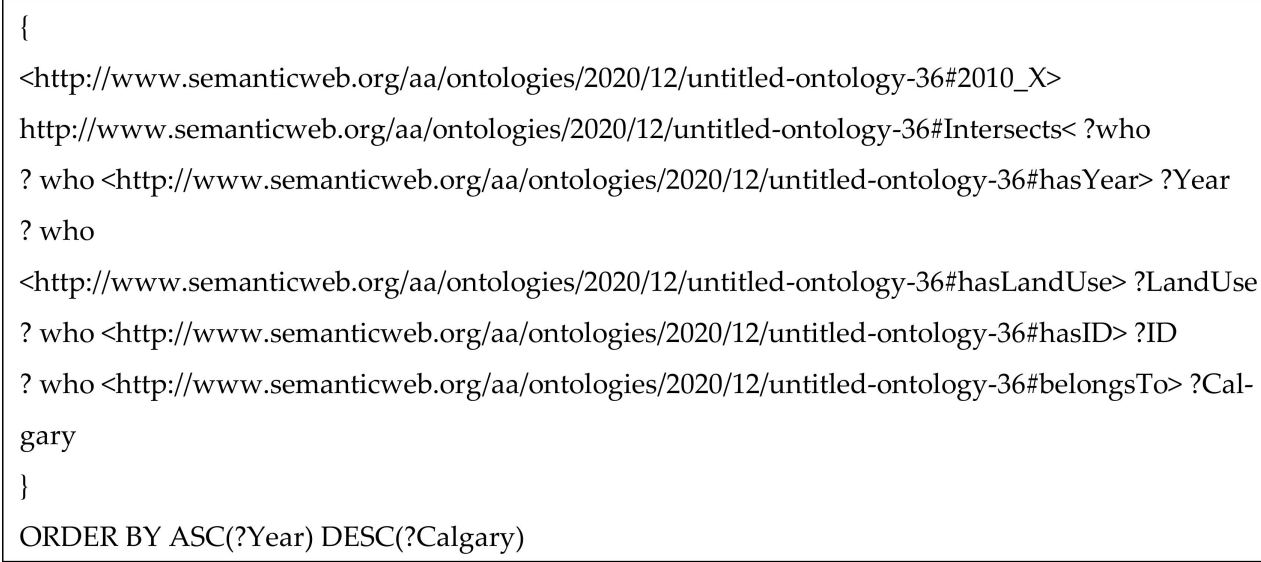

Figure 6. (a) Pseudo code of the semantic query. Example semantic query triplets using SPARQL: (b) the land use composition; (c) the semantic query for the land use change at the case study area (2010_X) from 2010 to 2015.

In the LUCO approach, an example, Triple, is defined by the following Table 3 statement:

Table 3. Triple example in the LUCO approach.

\begin{tabular}{lll}
\hline Subject & Predicate & Object \\
\hline Land use 2001 & HasGrowth & Land use 2010 \\
\hline
\end{tabular}

The above statement is an example of a Triple, using the HasGrowth relationship as a predicate. In order to represent the spatial association in time, this study defines a similar affiliation property in the LUCO model, which makes it possible to link two consecutive times. This Triple enabled it to link two consecutive times. The relations defined by Allen [42] were used to express the temporal constraints (Meets, Equal).

While the value range of a property (predicate) is a data type, such as numbers or time, supported by the OWL standard, they were defined as a data property. To test the LUCO, we designed a series of queries to detect the land use change. Then, the land use change data were used in neural network models to predict traffic.

To test the geospatial semantic, this study designed queries based on the LUCO model. The three queries were the spatial land use object's information, the historical trajectory, and the trend(s). The outcomes from LUCO are shown in Figure 6, which depicts the semantic geospatial queries for the land use change. In other words, the rules, present condition, and tendencies of any change in land use could be obtained using a combination of different properties in the LUCO by the OWL standard [43].

\subsubsection{Deep Neural Network: Recurrent Neural Network (RNN)}

To address traffic flow prediction, the Recurrent Neural Network deep learning methods, including land use change from LUCO, was proposed. Generally speaking, land use changes would affect the traffic flow. Initially, predictions were being made by only using time series data, but then it proceeded to incorporating land use changes.

Despite other predictor variables remaining the same, it was hypothesized that an inclusive land use variable would perform better to predict traffic. For their corresponding previous intervals, land use changes and traffic flow were added to both the training and testing data set. In the training stage, the land use changes positively impacted traffic flow so it could learn to reflect the flow under various land use conditions.

RNNs consider two types of input (i.e., the present and the recent past), which were combined to determine how the network should respond to new data. RNNs could efficiently manage sequence information. Figure 7 illustrates the basic and proposed concept of RNN using a function of inputs from previous time steps, also known as memory 
cells. RNNs were found to be flexible in their inputs and outputs for both sequences and single vector values.
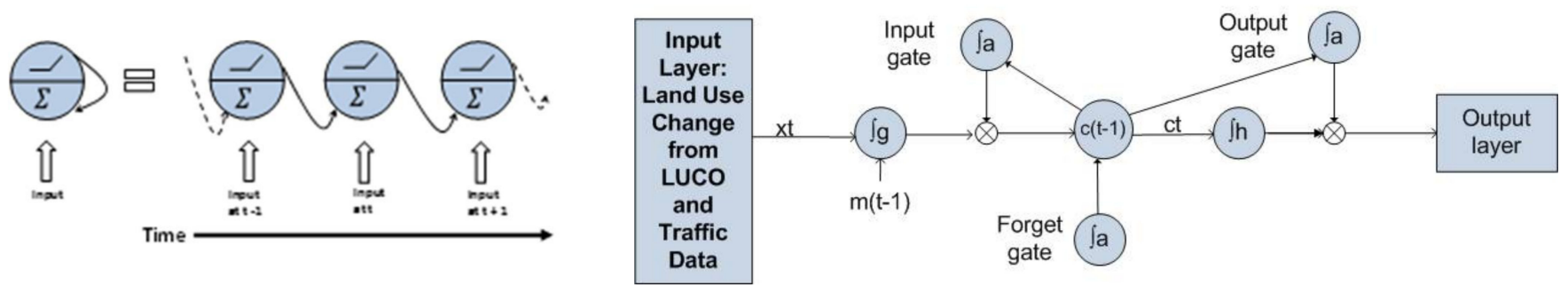

Figure 7. RNN overall concept (left) and proposed RNN (RNN-LSTM) (right).

When examining time series calculations, RNNs showed some gradient challenges when the model was run. To address these challenges, shortened time steps were attempted but results found a negative outcome on long-term predictions. Another issue that was found was, after a while, the network began to 'forget' the initial inputs. The LSTM (Long Short-Term Memory) cell was established to tackle this issue. This type of cell is a specific subgroup of RNN that was presented by Sak et al. [49] to bypass any long-term reliance on dependency problems.

We proposed a deep learning method for traffic flow prediction to learn the effective features of traffic flow and land use data. In this study, together with the traffic flow from previous intervals, the land use data for corresponding previous intervals was added to both the training and the testing data set. In the training stage, the feature of land use impact on traffic flow could be learned to reflect the land use change. Therefore, in the test stage, the model will have been better in its predictions when compared to those that neglected this land use feature [50]. The input data of the LSTM also contained the land use data for the previous corresponding intervals of the traffic data. This model had one hidden layer as the memory cell to recurrently save the time series' characteristics of the traffic data. The network trained by labeled data in a BP manner, using the gradient descent optimization method. The structure of the LSTM is shown in Figure 7.

The monthly data collected from two traffic monitoring stations over a 16-year period (2000-2015) were used for this study to explore the performance of the proposed model, as described in Section 3.1. The procedures used in the deep learning methodology are as follows:

Step 1. Use land use change data from LUCO and traffic data to prepare the training and test data sets.

Step 2. Use traffic data to forecast univariate time series traffic.

Step 3. Use traffic and land use change data to forecast multivariate time series traffic.

Step 4. Predict single-step and multi-step future data.

Step 5. Use the test data to examine the prediction performance. Compare the results with other models.

\subsubsection{Results Evaluation}

For the purposes of this study, Neural Network and ARIMA were compared in order to evaluate the RNN model's prediction usefulness. The different elements and performance of these models are defined by error calculations. The evaluation error metrics-Mean Absolute Error (MAE), Root Mean Squared Error (RMSE), and the Mean Absolute Percentage Error (MAPE) - were applied to address these error calculations.

Neural Network (ANN) was found to be an effective non-linear process that analyzes the interactions between the inputs and outputs. It focuses on the structure and function of biological neural networks [18]. The activation function of ANN had a non-linear transformation of input. 
ARIMA (Auto Regressive Integrated Moving Average) is a widely used statistical method for time series modeling. This model captured a complement of different standard structures in the time series data.

The root mean square error (RMSE), the mean absolute error (MAE), and the mean absolute percentage error (MAPE) were selected to evaluate the model's performance. The $\mathrm{MAE}$ is an indicator for the average deviation of the predicted values from corresponding observed values. MAE provided useful information on a model's performance; whereby, lower MAE values indicated improved model prediction. The Root Mean Squared Error (RMSE) measures the differences between values predicted by the model and the values observed. Lower RMSE values are an indication of a better prediction model. The expressions of all measures are provided in the formula below. The MAE and RMSE units were vehicles per day (veh/day).

$$
\begin{gathered}
M A E=\frac{1}{n} \sum_{i=0}^{n}\left|\overline{y_{i}}-y_{i}\right| \\
R M S E=\frac{\sum_{i=1}^{n}\left(\overline{y_{i}}-y_{i}\right)^{2}}{\sum_{i=1}^{n}\left(\operatorname{mean}(y)-y_{i}\right)^{2}} \\
M A P E=\frac{1}{\bar{n}} \sum_{i=0}^{n}\left|\overline{y_{i}}-y_{i}\right| / \overline{y_{i}}
\end{gathered}
$$

$\mathrm{N}(\mathrm{n})$ refers to the total number of points, while $\overline{y_{i}}$ and $y_{i}$ are the predicted values and their corresponding observations. This exact metric was used to evaluate the proposed model's accuracy with other predictive models adapted from Xu et al. [51].

Analysis of variance (ANOVA) was used to test the statistical significance of the modeling results. ANOVA estimated the variance within groups (original traffic data) and between each group (the model results). The following hypothesis was tested using ANOVA:

H0: Y1 (traffic prediction); Y2 (traffic prediction includes land use change from LUCO).

The equations above represent the average estimation obtained from the actual data model using traffic plus land use data, as well as traffic-only data. This hypothesis statement was tested using the level of significance assumed as $0.05(\mathrm{p}<0.05)$. The implementation of the traffic prediction algorithm was performed in Python, using Tensorflow as the backend.

\section{Experiment Results}

\subsection{Land Use Change and Ontology Results}

The experimental results are illustrated in this section. The land use classification results for 2001 and the land use data from 2010 and 2015 were used to compare the land use types in Calgary in terms of ratios and spatial distribution. As shown in Figure 8, when examining the original land use data from 2001, the derived land use map for 2010 revealed the same patterns, and the land use spatial distribution remained the same. For the land use ratio of the different categories in Table 4, all the five land use types were compared.

The map of the land use change for Calgary is shown in Figure 8. Within the study area, open spaces accounted for the largest amount, covering $70 \mathrm{~km}^{2}(55 \%)$. Figure 8 shows that open space was the dominant land use type in the surrounding regions. Among the residential areas, most of the regions were classified as "Residential Medium and Low". The resulting land use map discovered was composed of clusters of the same type of land use, which suggested that the proposed model was suitable for the large-scale monitoring of urban areas. Moreover, the land use ratio (Figure 9) also reflected the patterns by integrating the qualitative and quantitative information. As shown in Table 4 and Figure 9, there was a decrease in open space and an increase in the residential, industrial, and infrastructure areas when compared to 2001. Calgary experienced substantial growth in its residential areas and investment in municipal infrastructures and public services, such as roads. 

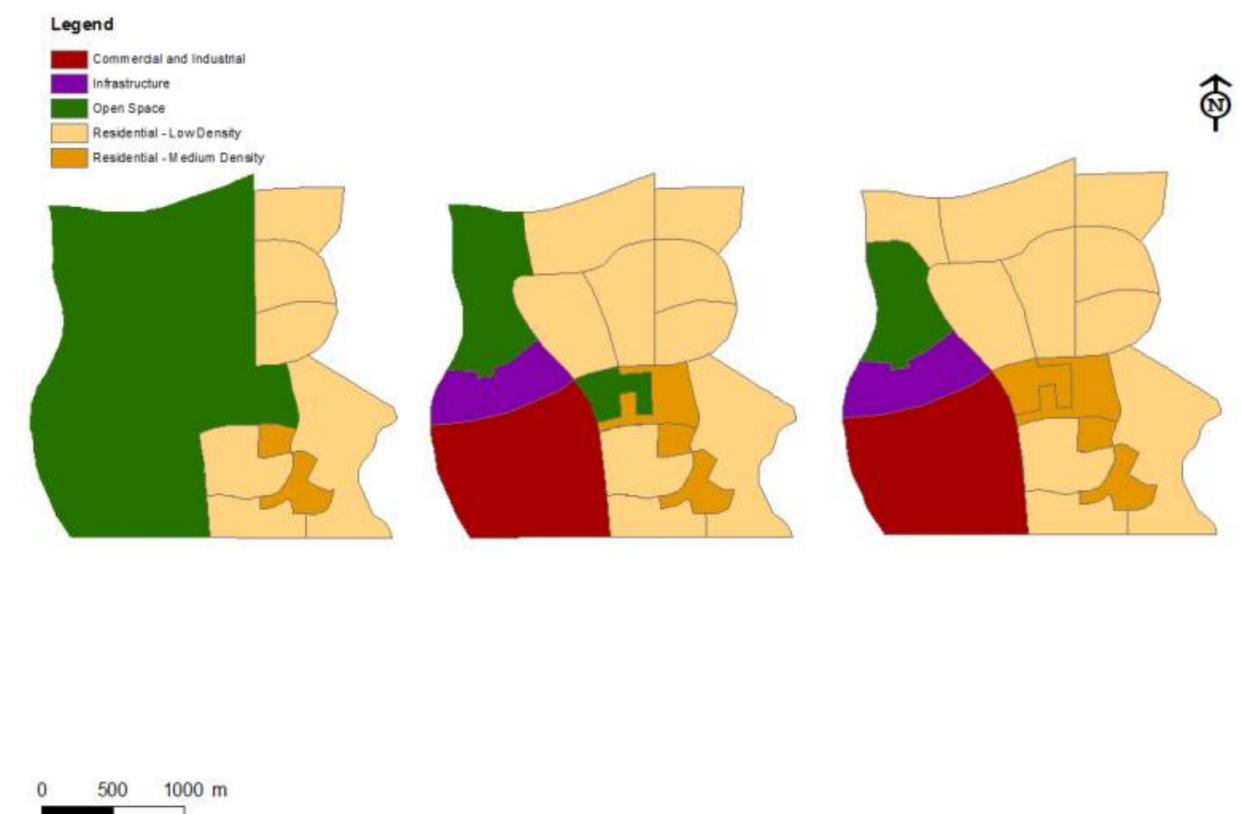

Figure 8. Land use change example: northeast Calgary. Left, 2001; Middle, 2010; Right 2015.

Table 4. Summary statistics of land use change (area in $\mathrm{km}^{2}$ ) between the years 2001, 2005, and 2015.

\begin{tabular}{lllllc}
\hline Land Use & Area 2001 & Area 2010 & Area 2015 & \% Change 2001 to 2010 & \% Change 2010 to 2015 \\
\hline Open Space & 70 & 55 & 40 & -20 & -27 \\
Major Infrastructure & 30 & 30 & 40 & 0 & 33 \\
Residential Low Density & 15 & 15 & 20 & 0 & 33 \\
Residential Medium Density & 5 & 10 & 10 & 100 & 0 \\
Commercial and Industrial & 0 & 10 & 10 & New land use in 2010 & 0 \\
\hline
\end{tabular}

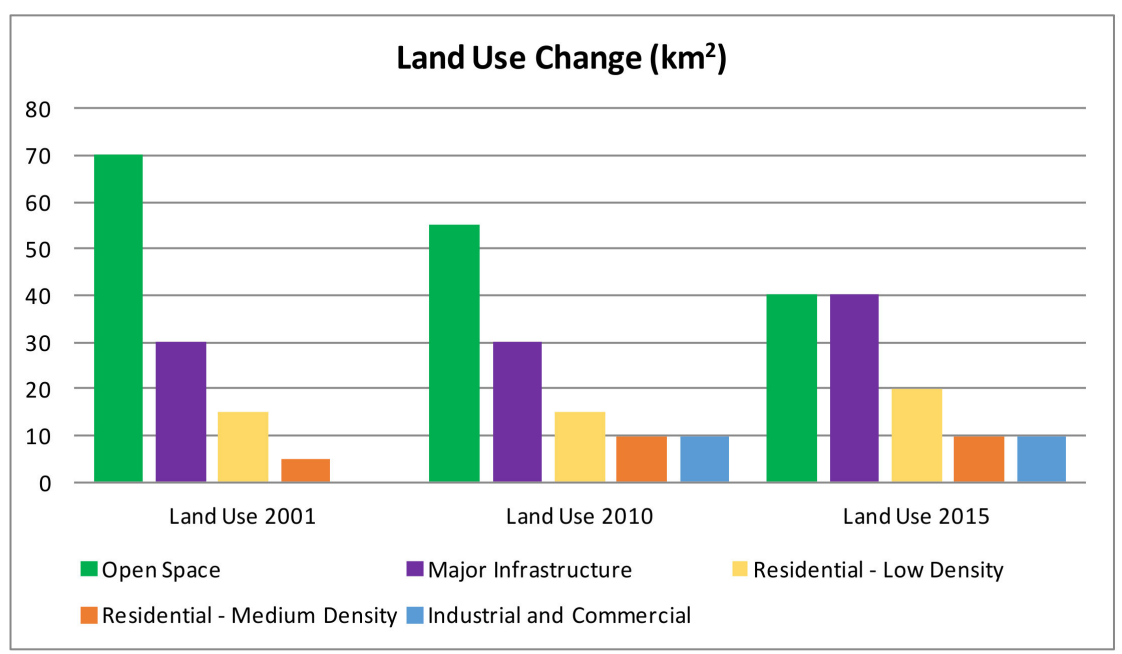

Figure 9. Land use change from LUCO.

Based on this LUCO, it was possible to derive an insightful interpretation to explain the situation. As noted in the Table 4, between 2001, 2010, and 2015, open space extended to connect the industrial or residential land uses. As the industrial or residential land use was more accessible to consumers, it was then expanded to an urbanization process. Overall, it was reasonably acceptable to obtain land use change results with the support of the LUCO model. The results were used to calculate the land use change from 2001 to 2010 and 2010 to 2015. Table 4 summarizes the computation of the areas using land use types. Two types of information can be retrieved from this LUCO model. On one hand, it could easily detect 
a gain or loss for each type of land use. On the other hand, it could determine increments in gains or losses that occurred within a given timeframe. This approach supported the validity of LUCO. The study area is considered to be a snapshot of multiple time segments, each belonging to a land use type. The next step was to explore further how land use changes affected the ability to predict traffic.

\subsection{Traffic Prediction Using RNN}

LUCO identified Open Space, Industrial, Residential, and Infrastructure as the key land use changes in northeast Calgary. These types of land uses were incorporated into traffic prediction models. As mentioned in Section 3.1, this study used traffic data collected from Calgary for the RNN model. All traffic data had connected types of sequences, i.e., each level was dependent upon the previous one, resulting in a Long Short-Term Memory (LSTM) model being incorporated.

This study applied multivariate time series traffic prediction modeling into a supervised learning format, similar to the method described in Jia et al. [50]. The traffic prediction model was created to learn the features in a historical time series dataset in order to make multi-step traffic flow predictions. The proposed framework incorporated an eight-layered LSTM auto-encoder architecture. For the interconnected layers, the activation function was used along with the Rectified Linear Unit (ReLU) function, which introduced non-linearity into the learning process. The performance of the deep learning networks was dependent on critical parameters that had to be predetermined, using a process known as hyperparameter optimization. To detect the optimal set of hyperparameters, a grid search framework was used.

To compare with other methods, the ANN and ARIMA models were selected to represent shallow machine learning and standard benchmarks. For ARIMA, the optimum structure was ARIMA $(4,1,2)$; this means it used a lag value of 4 for autoregression; it used a difference order of 1 to make the sequence stationary; and it used a moving average window of 2. Figure 10 shows the predicted and ground truth (actual) observations for the three prediction models, as described in Section 3.2. The x-axes in the figure represent the time, while the $y$-axes represent the sum of the traffic flows at the data collection points.

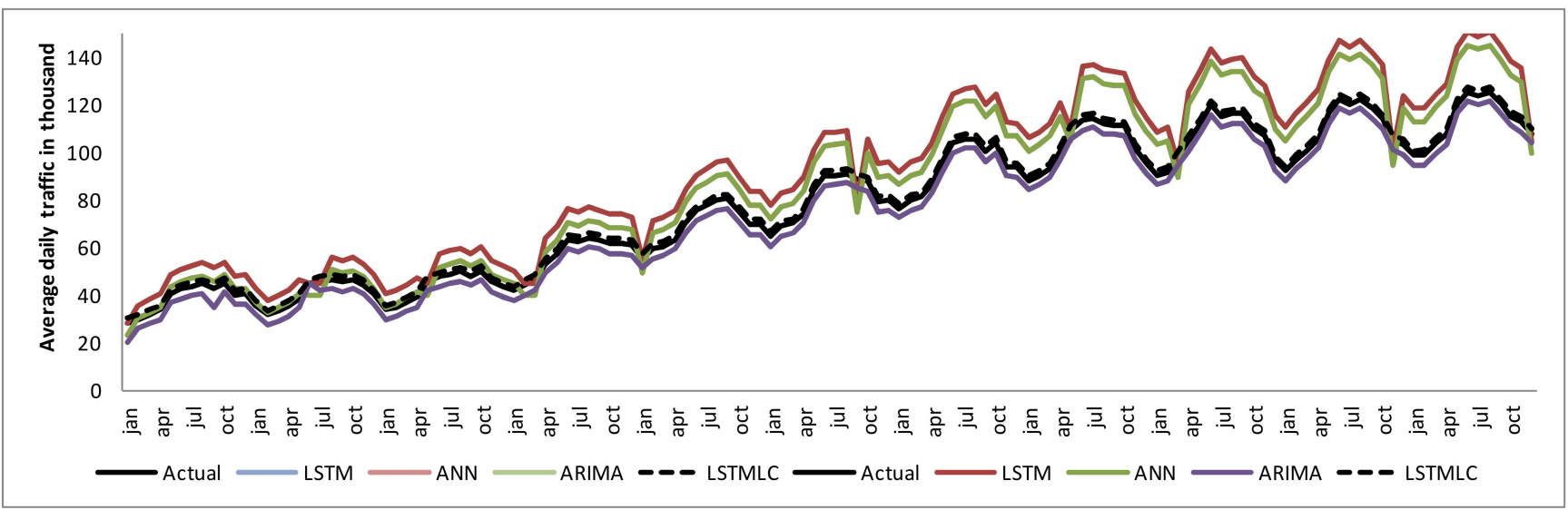

Figure 10. Observed vs. predicted model comparison.

The span of the test datasets used for this study ranged from January 1st, 2001 to December 31st, 2015. Table 5 shows the performance evaluation results for the proposed and baseline models. As the table demonstrates, there was a significant improvement in the predictive accuracy when land use datasets were included. It is also worth noting, the proposed model outperformed other baseline models. 
Table 5. Model results comparison.

\begin{tabular}{llll}
\hline Model & RMSE & MAE & MAPE \\
\hline LSTM LC & 6.87 & 6.54 & 0.19 \\
LSTM & 10.41 & 9.14 & 0.35 \\
ANN & 13.22 & 11.21 & 0.46 \\
ARIMA & 15.37 & 14.5 & 0.65 \\
\hline
\end{tabular}

The differences between the MAE, MAPE, and RMSE observed values and the predicted data are presented in Table 5. The models using land use and traffic data recorded the lowest MAE, RMSE, and MAPE. Similarly, Table 5 shows that the LSTMLC (LSTM that includes land use change) model performed competitively in contrast to the outcomes from the other models. The ARIMA model returned the poorest set of results; however, it had the shortest training time. The conclusion made from these outcomes is that there is a clearly demonstrated improvement in reducing errors when compared to the models using traffic and land use datasets. This led to a further conclusion that the inclusion of land use improved the prediction accuracy when combined with traffic and land use data.

Although there seemed to be a slight improvement in the observed accuracy, it is worth noting that the cost-benefit realized by a reduction in the MAE using the traffic and land use dataset was significant.

From the ANOVA, the Fcritical value was 2.60 and $\mathrm{F}$ was 7.30 ; therefore, $\mathrm{F}$ had a larger value than Fcritical. ANOVA results are demonstrated indicating that if $\mathrm{F}$ was greater than Fcritical, the null hypothesis would be rejected. This indicated that the corresponding results were significantly different.

The test data set noted earlier included land use change as a variable. For comparison, several other predictors were selected, including ANN and ARIMA. These two were also implemented as benchmarks. Comparative experiments were completed revealing that the proposed models provide superior methods for predicting traffic when land use changes from the LUCO model were included.

\section{Discussion}

This study proposes and implements a computational framework to support the LUCO and deep learning with regard to space and time. We first proposed a conceptual LUCO model to represent the spatiotemporal variation of land, which is essential knowledge to support various studies, such as urban and transportation planning. Then land use change results were used in the RNN to predict traffic. Based on the knowledge defined in the ontology and related reasoning, rules were developed to support the query and change trajectory reasoning of areas with land use change. Two types of information can be retrieved from this LUCO analysis: first, to detect a gain or loss for each of the land use; and second, to determine how much of a gain or loss occurs between two time segments. Urban planners and decision-makers need to have knowledge about the existing land use change and also complete and up-to-date information on traffic prediction. Another purpose of using LUCO and RNN in this study is to integrate various levels of data in LUCO and deep learning to obtain traffic prediction and the way it is changing because of land use.

In this study, we combined the land use change ontology and deep neural network for traffic prediction. We found that the LUCO allowed the development of spatial datasets in order to explore the land changes in northeast Calgary. Subsequently, this land use change was used to predict traffic in the RNN model. Land use change was analyzed using available data from 2001, 2010, and 2015. The analysis revealed that there was a significant $40 \%$ decrease in the open space values, which exposed concerns, as this is a result of more residential and developed industrial areas. A similar result can be found in other works [12], which can be attributed to urban growth [12].

The changing characteristics of land use affect the ability to manage, organize, and build new developments as well as effectively plan for increased transportation needs. In this regard, LUCO was able to establish relationships between the land use types and 
traffic prediction. Ontology was found to be very successful in comparing and measuring types of land use changes. It was not only measuring the changes, but can also support traffic prediction.

To our knowledge, this is the first study that has explored land use change ontology and neural networks for traffic prediction. The presented research sought to answer these questions: What was the land use change ontology, and how was this change applied through using different deep learning and data mining methods to represent traffic prediction? To address these questions, we developed LUCO and RNN models. To our knowledge, this is the first study that has combined these two approaches.

This paper introduced the progress and execution of a computational framework to support the modeling of land use change information with regard to space, time, and topology. The knowledge base that the system relied on was encoded in an ontology that modelled various properties (i.e., image, spatial, and temporal data). Related studies also suggested that land use change should be focused on space, time, and topology [10]. However, beyond this, modeling complexity increased dramatically when a change process involved combining multiple dimensions, such as traffic prediction from land use change.

The result obtained when using LUCO motivated further investigation of how the deep learning model performed when land use change was incorporated as the key variable. The study then compared the differences between RNN and other benchmark models for the prediction of time series traffic data. ANN and ARIMA models were proposed as benchmark models, and their performances in predicting time series data were compared. The experimental results, using the Calgary data set, indicated that the RNN could effectively discover the features of traffic flow under various land use change conditions. Additionally, the comparison showed deep learning with multisource data inputs is a promising field of study. These expected results were consistent with other researchers' works [29,33-35].

Unlike most of the spatio-temporal domain models using a relational database, the LUCO model was based on semantic technologies, which gave it the advantage of advanced modeling and analysis of the geospatial environment context. As a result, the machine learning was able to provide exceptional interpretations, which resulted in a superior amount of information and better understanding of the dynamics of the phenomena that was seen in previously proposed models.

The objective of this study was to incorporate land use change ontology with a deep neural network for traffic prediction. In terms of study limitations, the proposed methodology did not focus on providing a comparison of the differences between the land use change models. However, a couple of suggestions can be considered for future studies. First, different kinds of land use change models can be evaluated, such as GIS-based statistical modeling and evaluation using a semantic model. As a further step, the influence of neighbors in the land use change process can be analyzed through taking the adjacency relations between different kinds of lands into consideration.

In this paper, the urban traffic prediction model explored the effectiveness of integrating information obtained from land use changes. The baseline model proposed here employed a deep LSTM architecture, incorporating traffic flow parameters as well as land use. The work presented in this study was restricted to a single arterial road. This study did not consider a few other key variables, such as other roads, demographics, and weather. Although there are limitations, using the model as-is, i.e., reusing the proposed model architecture, could help account for additional roads in cities. For instance, the road, demographics, and weather can be added for the various locations under consideration, while retaining the model architecture and composition. On the other hand, it may be possible to consider the correlation among roads with some additional data features (i.e., how does an accident on road A affect road B?). Future work should focus on the integration of complementary entities of a city's roads and infrastructures. The proximity of these factors might have positive or negative impact on spatial-temporal transitions and filiation relationships. For instance, the model could be able to infer the impact of a new highway between communities or the influence of a city in neighbouring rural areas. 
Traffic planning and prediction processes can be challenging due to the non-linearity behavior inherent to these processes, e.g., real-world uncertainties, such as weather, accidents, and road maintenance, will be present. Besides urban growth and land use, changes might occur in combination with a series of decision-making processes, which are not deterministic but rather the result of competing local demands and uncontrolled processes [52]. Fuzzy approximation theory is ideally suited to treat the complexity and uncertainties in the decision-making process. This fuzzy approximation idea could be used as a complement for a future study on how fuzzy approximation can be applied to model land use change inherent to the traffic prediction process, since real world uncertainties, such as weather, roads maintenance, etc., can be considered.

The work in this study was restricted to one city in Canada. The proposed methods could be applied for use in making predictions in other cities with various data features, such as the evaluation of how an accident on a specific road can affect the traffic on other roads. Nevertheless, incorporating this model into a wide geographic region continues to present considerable challenges. These were demonstrated when there were substantial increases in the model's computational process and computational time. Larger geographical areas would require more data and a greater training time, which can lead to lower accuracy in its prediction. Future work should consider this issue. However, it is important to note there would be trade-offs, such as the need for greater time requirements in training and processing with the improvements in semantic analysis of land uses.

The proposed study demonstrated high performance for the application domain. However, this performance may vary in this domain when applied to other cities. Model performance under other cities might become inadequate or unsatisfactory because of different cities' uniqueness or scalability capabilities $[53,54]$. Methodologies should be adjusted according to the cities' scalability capability, database, land use types and other related conditions.

We performed experimental testing, and our results show that the LUCO and RNN exhibit good performance and have an indication value in the planning of land use, transportation, and their surroundings. This type of study can contribute to the construction of smart cities [55]. New technologies, such as LUCO and deep learning, are advancing, and smart cities can be made smarter by integrating the LUCO and deep learning, which connect and interact with humans, the environment, and smart devices [56]. This paper discussed selective technologies that can potentially contribute to developing an intelligent environment and smart cities. While the connectivity and efficiency of cities is important, the analysis of the impact of land development and traffic in the city is crucial to decision and policy makers. Future studies can address the question of how a smart city can be improved by utilizing these technologies.

The rapid development of the semantic web and exponential growth in the use of ontology, make new and different multi-dimensional, quality smart cities an opportunity, in which data is collected from various sources. As a result, in this paper, we develop the concept of LUCO and RNN, which can be considered in future studies of smart cities. Such an ontology and deep learning are beneficial for both citizens and city planners as it allows interoperability among different land use and transportation frameworks [57].

There are a wide range of technologies and applications available, but understanding their function, interoperability, and compatibility within a city requires more discussion around system designs and architecture. These aspects can be the basis of developing an agenda for future studies. In particular, using ontology and deep learning for advanced tools, such as Geospatial Artificial Intelligence, Unmanned Aerial Vehicles, Geospatial Augmented Reality apps, and Light Detection and Ranging, in smart cities need to be discussed [56].

As future work, the proposed study may be encoded in an ontology implementation language such as OWL (https:/ / www.w3.org/OWL accessed on 7 May 2021), and it may be submitted to the Ontology Design Pattern catalogue. Besides, these patterns are being implemented in ontology under development for smart cities. Moreover, future studies to reach an agreement with other patterns from domains not covered in this work may be an interesting avenue [58]. 
Finally, the nature of the ontology eases the integration of various spatial datasets. In future work, we plan to integrate heterogeneous data sources into our study. Additionally, we propose to implement and integrate the spatial ontology and reasoning framework into an operational infrastructure framework to support collaborative Land Use Change-Deep Learning Traffic Prediction querying and decision making.

\section{Conclusions}

In this paper, methods for analyzing land use change, using a semantic approach, were presented. The Land Use Change Ontology (LUCO) model allowed the development of spatial datasets in order to explore the land changes in northeast Calgary. Subsequently, this land use change was used to predict traffic in the Recurrent Neural Network model. Land use change was analyzed using data available and based on a calculated area of $120 \mathrm{~km}^{2}$. The analysis revealed a substantial decrease in open space and an increase in high residential and industrial land use types in northeast Calgary.

From the LUCO, further investigation was pursued as to how the deep learning model performed when land use change was used as the key variable. This study also aimed to compare the differences between RNN and other benchmark models for the prediction of time series traffic data. For this purpose, ANN and ARIMA models were proposed as the benchmark models. It was explored in terms of how it performed in predicting time series data. The experimental results, using the Calgary data set, indicated that the RNN could effectively discover the features of traffic flow under various land use change conditions. This work suggests that deep learning with multisource data inputs is a promising field of study. These integrated approaches to improve traffic prediction may lead to a more effective means of enhancing transportation planning and management.

Author Contributions: Conceptualization, Abul Azad and Xin Wang; methodology, Abul Azad; supervision, Xin Wang; writing-original draft, Abul Azad; writing-review and editing, Xin Wang. All authors have read and agreed to the published version of the manuscript.

Funding: This research received no external funding.

Data Availability Statement: Not applicable.

Acknowledgments: This work was supported by the Natural Sciences and Engineering Research Council of Canada Discovery Grant.

Conflicts of Interest: The authors declare no conflict of interest.

\section{References}

1. Calgary Still Canada's Fastest Growing Metropolitan Area Despite Downturn, Census Reveals; CBC News, The Canadian Press: Toronto, ON, Canada; Available online: https://www.cbc.ca/news/canada/calgary/calgary-census-data-growth-population-recession-1. 3972079 (accessed on 18 September 2020).

2. Duan, Y.; Lei, K.; Tong, H.; Li, B.; Wang, W.; Hou, Q. Land use characteristics of Xi'an residential blocks based on pedestrian traffic system. Alex. Eng. J. 2021, 60, 15-24. [CrossRef]

3. Sfyridis, A.; Agnolucci, P. Annual average daily traffic estimation in England and Wales: An application of clustering and regression modelling. J. Transp. Geogr. 2020, 83, 102658. Available online: https://ideas.repec.org/a/eee/jotrge/v83y2020ics096 669231930568x.html (accessed on 14 January 2021). [CrossRef]

4. Pulugurtha, S.S.; Kusam, P.R. Modeling AADT using integrated spatial data from multiple network buffer bandwidths. In Proceedings of the Transportation Research Board 91st Annual Meeting, Transportation Research Board, Washington, DC, USA, 22-26 January 2012.

5. Azad, A.; Wang, X. Prediction of Traffic Counts Using Statistical and Neural Network Models. Geomatica 2015, 69, 297-311. [CrossRef]

6. Aljoufie, M. Urban Growth and Transport in Jeddah: Dynamic Modeling and Assessement. Ph.D. Thesis, University of Twente, Ensched, The Netherlands, 2012; pp. 1-189.

7. De Pinho, C.M.D.; Fonseca, L.M.G.; Korting, T.S.; de Almeida, C.M.; Kux, H.J.H. Land-cover classification of an intra-urban environment using high-resolution images and object-based image analysis. Int. J. Remote Sens. 2012, 33, 5973-5995. [CrossRef]

8. Dimitrijevic, B. A Method for Assessing Transportation Impacts of New Land Developments Using Integrated Land Use and Transportation Network Modeling. Ph.D. Thesis, New Jersey Institute of Technology, Newark, NJ, USA, 2018. 
9. Rahman, M.L.; Baker, D.; Rahman, M.S.-U. Modelling induced travel demand in a developing country: Evidence from Dhaka, Bangladesh. Transp. Res. Procedia 2020, 48, 3439-3456. [CrossRef]

10. Montenegro, N.; Gomes, J.C.; Urbano, P.; Duarte, J.P. A land use planning ontology: LBCS. Future Internet 2012, 4, 65-82. [CrossRef]

11. Li, W.; Zhou, X.; Wu, S. An Integrated Software Framework to Support Semantic Modeling and Reasoning of Spatiotemporal Change of Geographical Objects: A Use Case of Land Use and Land Cover Change Study. Isprs Int. J. Geo-Inf. 2016, 5, 179. [CrossRef]

12. Harbelot, B.; Arenas, H.; Cruz, C. LC3: A spatio-temporal and semantic model for knowledge discovery from geospatial datasets. Web Semant. Sci. Serv. Agents World Wide Web 2015, 35, 76-85. [CrossRef]

13. Epstein, B. Social Ontology. In The Stanford Encyclopedia of Philosophy; Zalta, E.N., Ed.; Metaphysics Research Lab, Stanford University: Stanford, CA, USA, 2018.

14. Studer, R.; Benjamins, R.; Fensel, D. Knowledge Engineering: Principles and Methods. Data Knowl. Eng. 1998, 25, 161-197. [CrossRef]

15. Castro, P.S.; Zhang, D.; Li, S. Urban traffic modelling and prediction using large scale taxi GPS traces. Pervasive 2012, 7319, 57-72.

16. Giannotti, F.; Nanni, M.; Pedreschi, D.; Pinelli, F. Trajectory pattern analysis for urban traffic. In Proceedings of the Second International Workshop on Computational Transportation Science (IWCTS), Seattle, WA, USA, 3 November 2009 ; pp. $43-47$.

17. Viktorović, M.; Yang, D.; de Vries, B. Connected Traffic Data Ontology (CTDO) for Intelligent Urban Traffic Systems Focused on Connected (Semi) Autonomous Vehicles. Sensors 2020, 20, 2961. [CrossRef] [PubMed]

18. Agatonovic-Kustrin, S.; Beresford, R. Basic concepts of artificial neural network (ANN) modeling and its application in pharmaceutical research. J. Pharm. Biomed. Anal. 2000, 22, 717-727. [CrossRef]

19. Schmidhuber, J. Deep learning in neural networks: An overview. Neural Netw. 2015, 61, 85-117. [CrossRef] [PubMed]

20. Yu, H.; Liu, X.; Kong, B.; Li, R.; Wang, G. Landscape ecology development supported by geospatial technologies: A review. Ecol. Inform. 2019, 51, 185-192. [CrossRef]

21. Gomes, J.; Montenegro, N.; Urbano, P. A land use identification and visualization tool driven by OWL ontologies. CEUR Workshop Proceedings. 2012. Available online: https:/ / www.researchgate.net/publication/268436391_A_Land_Use_Identification_and_ Visualization_Tool_Driven_by_OWL_Ontologies/citation/download (accessed on 10 September 2020).

22. Hyvönen, E.; Tuominen, J.; Kauppinen, T.; Väätäinen, J. Representing and Utilizing Changing Historical Places as an Ontology Time Series. In Geospatial Semantics and the Semantic Web: Foundations, Algorithms, and Applications; Ashish, N., Sheth, A.P., Eds.; Springer: Boston, MA, USA, 2011; pp. 1-25.

23. Yang, L.; van Dam, K.H.; Majumdar, A.; Anvari, B.; Ochieng, W.Y.; Zhang, L. Integrated design of transport infrastructure and public spaces considering human behavior: A review of state-of-the-art methods and tools. Front. Archit. Res. 2019, 8, 429-453. [CrossRef]

24. Balestrieri, M.; Ganciu, A. The Use of Graphs to Explore the Network Paradigm in Urban and Territorial Studies; Springer: Cham, Switzerland, 2020. [CrossRef]

25. Deakin, E. Land Use and Transportation Planning in Response to Congestion Problems: A Review and Critique. Transp. Res. Rec. $1989,1237,10$.

26. Van der Voort, M.; Dougherty, M.; Watson, S. Combining Kohonen maps with ARIMA time series models to forecast traffic flow. Transp. Res. Part C Emerg. Technol. 1996, 4, 307-318. [CrossRef]

27. Okutani, I.; Stephanedes, Y.J. Dynamic prediction of traffic volume through Kalman filtering theory. Transp. Res. Part B Methodol. 1984, 18, 1-11. [CrossRef]

28. Nikravesh, Y.; Ajila, S.A.; Lung, C.; Ding, W. An experimental investigation of mobile network traffic prediction accuracy. Serv. Trans. Big Data 2016, 3, 1-16. [CrossRef]

29. Liu, Y.; Wang, Y.; Yang, X.; Zhang, L. Short-term travel time prediction by deep learning: A comparison of different LSTMDNN models. In Proceedings of the 2017 IEEE 20th International Conference on Intelligent Transportation Systems (ITSC), Yokohama, Japan, 16-19 October 2017.

30. Ateeq, M.; Ishmanov, F.; Afzal, M.K.; Naeem, M. Predicting delay in IoT using deep learning: A multiparametric approach. IEEE Access 2019, 7, 62022-62031. [CrossRef]

31. Hua, Y.; Zhao, Z.; Chen, X.; Li, R.; Zhang, H. Traffic prediction based on random connectivity in deep learning with long short-term memory. In Proceedings of the 2018 IEEE 88th Vehicular Technology Conference, Chicago, IL, USA, 27-30 August 2018.

32. Zhang, W.; Yu, Y.; Qi, Y.; Shu, F.; Wang, Y. Short-term traffic flow prediction based on spatio-temporal analysis and CNN deep learning. Transp. A Transp. Sci. 2019, 15, 1688-1711. [CrossRef]

33. Zhou, J.; Chang, H.; Cheng, X.; Zhao, X. A multiscale and high-precision LSTM-GASVR short-term traffic flow prediction model. Complexity 2020, 2020, 1434080. Available online: https://www.hindawi.com/journals/complexity/2020/1434080/ (accessed on 20 January 2021).

34. Wu, Q.; Fu, Q.; Nie, M. Graph wavelet long short-term memory neural network: A novel spatial-temporal network for traffic prediction. J. Phys. Conf. Ser. 2020, 1549, 042070. [CrossRef]

35. Narmadha, S.; Vijayakumar, D.V. Multivariate time series traffic prediction using long short term memory network. Int. J. Sci. Technol. Res. 2020, 9, 1-6.

36. Data-W3C. Available online: https://www.w3.org/standards/semanticweb/data (accessed on 21 January 2021).

37. Maier, A.; Schnurr, H.-P.; Sure, Y. Ontology-Based Information Integration in the Automotive Industry. In The Semantic Web-ISWC 2003; Springer: Berlin/Heidelberg, Germany, 2003; pp. 897-912. [CrossRef] 
38. Hwang, S. Ontology-Based Spatial Clustering Method: Case Study of Traffic Accidents. 2003. Available online: http: / / ac.els-cdn.com/S0268401214001066/1-s2.0-S0268401214001066-main.pdf?_tid=bf539186-130b-11e7-8e34-00000aab0f27\& acdnat=1490632863_e211292f2483a9279f41e7132929b8f0 (accessed on 25 January 2021).

39. "Calgary," Wikipedia. 2020. Available online: https:/ / en.wikipedia.org/w / index.php?title=Calgary\&oldid=979293914 (accessed on 19 September 2020).

40. Brownlee, I. How To Backtest Machine Learning Models for Time Series Forecasting. Machine Learning Mastery. 2016. Available online: https://machinelearningmastery.com/backtest-machine-learning-models-time-series-forecasting/ (accessed on 21 January 2021).

41. Di Fonzo, T.; Marini, M. On the Extrapolation with the Denton Proportional Benchmarking Method. In IMF Working Papers; International Monetary Fund: Washington, DC, USA, 2012; Volume 12. Available online: https:/ /www.elibrary.imf.org/view/ journals /001/2012/169/001.2012.issue-169-en.xml (accessed on 25 January 2021). [CrossRef]

42. Grüninger, I.; Li, Z. The time ontology of Allen's interval algebra. In Proceedings of the 24th International Symposium on Temporal Representation and Reasoning, Mons, Belgium, 16-18 October 2017; pp. 1-16. [CrossRef]

43. OWL-Semantic Web Standards. Available online: https:/ / www.w3.org/OWL/ (accessed on 21 January 2021).

44. Object-Based Image Analysis. Available online: https://www.gim-international.com/content/article/object-based-imageanalysis (accessed on 21 January 2021).

45. Drăguț, L.; Blaschke, T. Automated classification of landform elements using object-based image analysis. Geomorphology 2006, 81, 330-344. [CrossRef]

46. Booth, B.; Mitchell, A. Getting Started with ArcGIS; ESRI Press: Redlands, CA, USA, 2001; pp. 1-260; ISBN 101879102935.

47. Appiah, D.O.; Schröder, D.; Forkuo, E.K.; Bugri, J.T. Application of Geo-Information Techniques in Land Use and Land Cover Change Analysis in a Peri-Urban District of Ghana. ISPRS Int. J. Geo-Inf. 2015, 4, 1265. [CrossRef]

48. McBride, B. Jena: A semantic Web toolkit. IEEE Internet Comput. 2002, 6, 55-59. [CrossRef]

49. Sak, H.; Senior, A.; Beaufays, F. Long short-term memory based recurrent neural network architectures for large vocabulary speech recognition. arXiv 2014, arXiv:1402.1128.

50. Jia, Y.; Wu, J.; Xu, M. Traffic flow prediction with rainfall impact using a deep learning method. J. Adv. Transp. 2017, 722, 1-10. [CrossRef]

51. $\mathrm{Xu}, \mathrm{W}$; Wang, Q.; Chen, R. Spatio-temporal prediction of crop disease severity for agricultural emergency management based on recurrent neural networks. GeoInformatica 2018, 22, 363-381. [CrossRef]

52. Al-Ahmadi, K. Modelling Driving Forces of Urban Growth with Fuzzy Sets and GIS. In Spatial Analysis, Modelling and Planning Rocha, K., Tenedório, J.A., Eds.; IntechOpen: Rijeka, Croatia, 2018; Chapter 7. Available online: https://www.intechopen.com/ books/spatial-analysis-modelling-and-planning/modelling-driving-forces-of-urban-growth-with-fuzzy-sets-and-gis (accessed on 15 January 2021). [CrossRef]

53. Gómez, J.A.; Patiño, J.E.; Duque, J.C.; Passos, S. Spatiotemporal Modeling of Urban Growth Using Machine Learning. Remote Sens. 2020, 12, 109. [CrossRef]

54. Liu, L.; Silva, E.A.; Wu, C.; Wang, H. A machine learning-based method for the large-scale evaluation of the qualities of the urban environment. Comput. Environ. Urban Syst. 2017, 65, 113-125. [CrossRef]

55. Ma, Y.; Liu, S.; Xue, G.; Gong, D. Soft Sensor with Deep Learning for Functional Region Detection in Urban Environments. Sensors 2020, 20, 3348. [CrossRef] [PubMed]

56. Shirowzhan, S.; Tan, W.; Sepasgozar, S.M.E. Digital Twin and CyberGIS for Improving Connectivity and Measuring the Impact of Infrastructure Construction Planning in Smart Cities. Isprs Int. J. Geo-Inf. 2020, 9, 240. [CrossRef]

57. Abid, T.; Zarzour, H.; Laouar, M.R.; Khadir, M.T. Towards a smart city ontology. In Proceedings of the 2016 IEEE/ACS 13th International Conference of Computer Systems and Applications (AICCSA), Agadir, Morocco, 29 November-2 December 2016; pp. 1-6. [CrossRef]

58. Espinoza-Arias, P.; Poveda-Villalón, M.; García-Castro, R.; Corcho, O. Ontological Representation of Smart City Data: From Devices to Cities. Appl. Sci. 2019, 9, 32. [CrossRef] 